www.nature.com/bmt

\title{
Editorial
}

\section{Mini-allografts: ongoing trials in humans}

\author{
AM Carella ${ }^{1}$, R Champlin ${ }^{2}$, S Slavin ${ }^{3}$, P McSweeney ${ }^{4}$ and R Storb ${ }^{4}$ \\ ${ }^{1}$ San Martino Hospital, Genoa, Italy; ${ }^{2}$ MD Anderson Cancer Center, Houston, TX, USA; ${ }^{3}$ Hadassah University Hospital, Jerusalem, \\ Israel; and ${ }^{4}$ Fred Hutchinson Cancer Research Center, Seattle, WA, USA
}

\section{Summary:}

Conventional allogeneic stem cell transplantation is a valuable approach to therapy for many hematologic malignancies. However, high-dose conditioning regimens designed both to control the malignancy and to prevent graft rejection are associated with a high incidence of acute and long-term side-effects. This has largely precluded the use of allografting for patients older than 55 years or for younger patients with certain preexisting organ damage. In order to manage the sideeffects, transplants have traditionally been delivered in highly specialized hospital wards or intensive care settings. Thus, an important goal is to develop safer allografting procedures that can be extended to older patients or patients with pre-existing organ dysfunction who are currently excluded from consideration for transplant. Recent observations have shown that donor lymphocyte infusions (DLI) can eradicate some malignancies that relapse after conventional allografting. These observations confirmed earlier evidence in favor of a graft-versus-leukemia effect based on the association of graft-versus-host disease (GVHD) with a lower likelihood of relapse of malignancy after allografting. Given the potential efficacy of DLI as the sole modality for eradication of malignancy, new strategies for allografting can incorporate the concept of less intensive conditioning therapy which is given with the sole aim of facilitating allogeneic engraftment. Recent pre-clinical studies in a canine model have shown that conditioning regimens for allografting can be markedly reduced in intensity yet still achieve the goal of engraftment. This review briefly summarizes the initial translational clinical studies, using a minimally myelosuppressive-conditioning regimen based on low dose total body irradiation (TBI) or fludarabine alone or in combination with other drugs followed by a short course of immunosuppression with post-grafting cyclosporine and methotrexate or mycophenolate mofetil. Bone Marrow Transplantation (2000) 25, 345-350.

Keywords: allografting; nonmyeloablative regimens; graft-versus-tumor

Correspondence: Prof A Carella, Dept Haematology, Ospedale San Martino, Largo Rosanno Brazzi, 10, 15142 Genova, Italy Received 1 September 1999; accepted 9 November 1999
Until the late 1970s it was thought that the mechanism of cure of a malignancy was due entirely to the conditioning therapy used before transplantation, and that the transplant itself was merely a supportive measure designed to allow the patient to receive myeloablative treatment without experiencing permanent aplasia. ${ }^{1,2}$ However, the desirable effects of conditioning regimens are offset by their high incidence of acute and long-term side-effects which are responsible for considerable transplant-related mortality. It is now known that the results of allogeneic hematopoietic stem cell transplantation (allo-SCT) are partly attributable to an immune effect mediated by donor lymphocytes, recognized as the graft-versus-leukemia (GVL) effect. There are major differences among malignancies in their susceptibility to GVL effects at least as judged by their response to DLI in the case of relapse after transplantation. Among the major leukemias treated with allo-SCT, CML is most sensitive, AML is intermediate and ALL is least affected by GVL. Indolent lymphoid malignancies (low grade lymphoma, CLL and myeloma) also appear to be susceptible to graft-versus-tumor effects. ${ }^{3-6} \mathrm{~A}$ major question is whether graft-versus-tumor effects are active against solid tumors. Pilot studies in breast cancer have reported antitumor responses in patients with graft-versus-host disease (GVHD) suggesting a graft-versus-adenocarcinoma effect. $^{7-9}$ Anecdotal data also suggest graft-versus-tumor effects against melanoma and potentially other malignancies. Further studies are required to determine whether a clinically meaningful graft-versus-tumor effect will occur in order to justify the added morbidity related to allo-SCT.

The success of DLI in inducing remissions in patients in relapse after allo-SCT suggests that long-term disease control may be feasible without high-dose induction therapy. ${ }^{10-18}$ Thus the emphasis has now switched to attempting to develop safer allografting procedures that can be extended to older patients and patients with pre-existing organ dysfunction who would not be eligible for conventional alloSCT. This approach reduces drug toxicity and may also reduce the risk for acute GVHD, since the clinical manifestations of this complication may partly result from the toxicity of the preparative regimen and subsequent cytokine release in addition to the alloreactivity of the graft. ${ }^{19}$

Non-myeloablative regimens followed by transfusion of allogeneic progenitor cells have been investigated in a number of centers as a way of harnessing the graft-versus-tumor effect. Initial experiences confirmed that such an approach is feasible in patients with hematologic neoplasia and gen- 
etic diseases. It is hoped that the incorporation of more effective but less toxic agents in conditioning regimens will reduce procedure-related morbidity and mortality and ultimately improve long-term outcomes for patients undergoing allo-SCT.

\section{The general concept}

The basic observation which serves as the rationale for nonmyeloablative stem cell transplantation originates from the demonstration that adoptive transfer of alloreactive donor lymphocytes can eradicate resistant malignant host cells that escaped maximally tolerated doses of chemoradiotherapy, an observation that has provided an option for cure of patients with a large variety of hematologic malignancies $^{10,11}$ especially CML. ${ }^{10-17}$ The observation that nonmyeloablative stem cell transplantation regimens based on low-dose total body irradiation (TBI) ${ }^{20}$ or fludarabine combined with cytotoxic agents have resulted in engraftment of allogeneic cells in hematologic malignancies, raises the possibility that such conditioning might even be useful in achieving a graft-versus-tumor effect. ${ }^{21,22}$ Fludarabine or low-dose TBI are crucial to these less intensive regimens. Fludarabine is an antileukemic and effective immunosuppressive agent as recently established at the MD Anderson Hospital in Houston. ${ }^{23}$ Nonmyeloablative chemotherapy using fludarabine combined with cytotoxic drugs clearly provides enough immunosuppression to allow engraftment of allogeneic blood progenitor cells. ${ }^{24}$ As recently reported, ${ }^{24}$ these nonmyeloablative approaches can be roughly divided into three categories: (1) reduced-intensity allo-SCT regimens; (2) pre-SCT host immunosuppression combined with post-SCT immunosuppression directed at both host and donor immune cells; and (3) high-intensity auto-SCT followed by an immunosuppressive cytotoxic allo-SCT regimen alone. In all these settings, allo-SCT is the platform for subsequent adoptive immunotherapy of the underlying malignancies using donor lymphocytes.

\section{Reduced-intensity allotransplantation regimens}

\section{Focusing on myelotoxic therapy}

Purine analog containing non-myeloablative chemotherapy is sufficiently immunosuppressive to allow engraftment of HLA compatible hematopoietic progenitor cells, and extended remissions were observed in some patients with $\mathrm{CML}^{21}$ or recurrent AML. ${ }^{25}$ Giralt et $a l^{26}$ reported a study combining melphalan $\left(180 \mathrm{mg} / \mathrm{m}^{2}\right)$ and either fludarabine $\left(125 \mathrm{mg} / \mathrm{m}^{2}\right)$ or cladribine $\left(60 \mathrm{mg} / \mathrm{m}^{2}\right)$ for treatment of advanced acute leukemia; patients with refractory or relapse usually recurred rapidly, but $56 \%$ of patients with chemotherapy-sensitive disease remained in continuous remission beyond 1 year. Indolent lymphoid malignancies also appear to be amenable to this strategy. Khouri et $a l^{27}$ treated 15 heavily pretreated patients with CLL or lymphoma using a nonmyeloablative regimen of fludarabine/cyclophosphamide or fludarabine/cytarabine/cisplatin. All patients had failed to respond or relapsed after primary chemo- therapy. Nine patients had CLL in relapse after prior fludarabine treatment and six had lymphoma. Eleven of the 15 patients had durable engraftment, with 50-100\% donor cells at 1 month post transplant, typically converting to $100 \%$ over the next 2 months spontaneously or after infusion of additional donor lymphocytes. Hematopoietic recovery was prompt and, with the exception of a patient with hepatitis $\mathrm{C}$ infection, no patient had non-hematologic toxicity of greater than grade 2 . The patients failing to engraft recovered endogenous hematopoiesis promptly and had no serious adverse effects. All 11 patients with engraftment have responded and eight have achieved complete remission. Maximal responses were slow to develop and gradually occur over a period of several months to 1 year.

The strategy of a nonablative preparative regimen was also applied to multiple myeloma to harness a graft-versusmyeloma effect while reducing regimen-related toxicities. The same team in Houston explored a regimen of melphalan $\left(140 \mathrm{mg} / \mathrm{m}^{2}\right)$ and fludarabine $\left(30 \mathrm{mg} / \mathrm{m}^{2}\right.$ for 4 days). This appears to be a promising strategy since seven of 13 patients with far advanced myeloma have achieved complete remissions. In conclusion, according to the Houston experience, the immune graft-versus-tumor effect is responsible for much of the benefit of allo-SCT. Use of less toxic, nonmyeloablative preparative regimens produced engraftment and generated graft-versus-tumor effects. This approach allowed the use of allo-SCT for older patients and those with comorbidities which precluded high-dose chemoradiotherapy. A regimen comparable to the one reported from Houston and consisting of cyclophosphamide/fludarabine was used with success by investigators in Bethesda. ${ }^{28}$

Investigators in Boston used CY 150 to $200 \mathrm{mg} / \mathrm{kg}$ along with ATG and thymic irradiation before HLA-identical sibling BMT in 21 patients with advanced, refractory hematologic malignancies. Grade II-IV GVHD was seen in only one of 21 patients not receiving DLI. Prophylactic DLI was given to patients in whom no GVHD was present by day 35 . Seven patients were alive and free of disease progression 105-548 (median 445) days after transplantation. Durable chimerism has been seen in about $90 \%$ of patients receiving HLA-identical and mismatched transplants with this protocol, and lasting mixed chimerism has been demonstrated $>1.5$ years in an extensively mismatched transplant recipient. ${ }^{29}$ The sustained remissions obtained in this group of patients with advanced and refractory disease suggest that this is a promising approach to achieving disease eradication, possibly with less GVHD than is seen with conventional transplants.

\section{Focusing on immunosuppressive therapy pre- transplantation}

Investigators at the Hadassah University Hospital in Jerusalem focused on induction of a window of immunosuppression (step 1) followed by induction of host-versus-graft tolerance accompanied by GVL effects mediated by donor lymphocytes infused with mobilized blood stem cells (step 2) or DLI given later as an outpatient procedure (step 3), reasoning that the same approach may offer the prospect 
of safer treatment of malignant and nonmalignant diseases in all age groups with minimal and controllable early and late procedure-related toxicity and minimal mortality. ${ }^{30,31}$ Intensive pre-transplant immunosuppressive therapy was accomplished with a combination of fludarabine 30 $\mathrm{mg} / \mathrm{m}^{2} /$ day for 6 days, busulfan $4 \mathrm{mg} / \mathrm{kg} /$ day for 2 days and anti-T lymphocyte globulin (ATG, Fresenius) 5-10 $\mathrm{mg} / \mathrm{kg} /$ day for 4 days. Following the conditioning, each patient received one or two infusions of G-CSF-mobilized blood stem cell collections. Low-dose cyclosporine (CsA) ( $3 \mathrm{mg} / \mathrm{kg} /$ day) was used as the sole GVHD prophylaxis for $<100$ days.

The most recent report included the cumulative experience with nonablative preparative regimen in 77 patients, of whom 63 patients had a variety of hematologic malignancies including CML, AML, ALL, resistant lymphomas and multiple myeloma and 14 had non-malignant diseases. The results of this study indicated that cure of otherwise lethal leukemia became feasible while minimizing early and late procedure-related toxicities and mortality, thus enabling safer cure for infants and children without impairment of growth and development. Similarly, nonmyeloablative SCT was well tolerated by elderly individuals, who were excluded until recently from BMT programs due to increased risk of procedure-related toxicity and mortality. Nonmyeloablative SCT resulted in durable engraftment in all 11 patients receiving bone marrow or blood stem cell allografts from matched unrelated donors with relatively low incidence of GVHD. ${ }^{32}$ Likewise, this less intensive conditioning regimen was well-tolerated by recipients of second stem cell allografts after failing a primary procedure of autologous bone marrow or blood SCT, suggesting that this regimen may be able to cure patients with resistant disease who have failed maximal tolerated doses of chemoradiotherapy. Moreover, replacement of host with donor immunohematopoietic cells and eradication of malignant or genetically abnormal stem cells and their products may be accomplished with no support of blood products, with no episodes of septic fever, while avoiding a nadir of aplasia, in $10-25 \%$ of the patients, respectively, in contrast to the pattern of recovery of hematopoiesis following standard myeloablative conditioning, hence possible on an outpatient basis.

\section{Allografting in an ambulatory care setting using immunosuppressive, minimally myelosuppressive conditioning}

Canine studies have been used in Seattle for several decades to study immunosuppressive regimens for achieving engraftment across minor and major histocompatibility barriers and for the prevention of GVHD. ${ }^{33}$ An advantage of this model is the ready availability of dog lymphocyte antigen (DLA)-identical littermates as a model for HLA-identical transplants in humans. The following allografting studies deal exclusively with the use of DLA-identical marrow grafts.
TBI delivered in a single fraction at a dose rate of $7 \mathrm{cGy}$ per minute in dogs given intensive supportive care but no stem cell grafts invariably caused lethal marrow failure at $\leqslant 400$ cGy whereas at doses $<200$ cGy dogs spontaneously recovered after a period of myelosuppression. ${ }^{34}$

\section{Enhancement of engraftment with post-graft immunosuppressive agents}

Single dose TBI at 920 cGy was sufficiently immunosuppressive to allow stable engraftment of marrow from $95 \%$ of DLA-identical littermate donors in the absence of postgraft immunosuppression. ${ }^{35}$ At 450 cGy, only $41 \%$ of dogs had stable engraftment. Additional experiments evaluated whether immunosuppressive agents given after transplant could facilitate engraftment. CsA alone was effective at a dose of 450 cGy with stable engraftment in seven of seven dogs, ${ }^{36}$ and the combination of CsA and mycophenolate mofetil (MMF) allowed for stable engraftment in 10 of 11 dogs after a non-myeloablative dose of only $200 \mathrm{cGy}$ TBI. ${ }^{37}$ Engraftment was achieved as stable mixed chimerism with the donor component comprising $45-80 \%$ of hematopoietic cells. Dogs were followed for up to several years and continued to show stable donor engraftment. At 100 cGy TBI, MMF and CsA did not allow for stable engraftment.

\section{Mechanism of low dose TBI}

To evaluate the mechanism by which low dose TBI contributed to engraftment six dogs were given marrow grafts after 450 cGy lymph node irradiation at $200 \mathrm{cGy} / \mathrm{min}$ and then post grafting MMF and $\mathrm{CsA} .{ }^{20}$ The radiation was given to target cervical, thoracic and upper abdominal lymph nodes while shielding the remainder of the dogs including most marrow spaces with lead blocks. Each dog showed evidence of initial mixed chimerism. Two dogs rejected their grafts, one died with full engraftment from GVHD, and three remained stable mixed chimeras with follow-up of 12 years. Marrow samples from unirradiated bones showed chimerism from as early as 4 weeks post transplant that persisted for the period of observation. These results supported the hypothesis that marrow grafts could create their own space and that myelosuppressive therapy was not necessary to establish allogeneic engraftment. These observations, together with results of additional preclinical studies involving second $\mathrm{T}$ cell activation signal blockade, ${ }^{38}$ suggest that in the future TBI may be replaced with immunosuppressive agents that lack the undesirable sideeffects of ionizing radiation.

\section{Clinical studies}

\section{Nonmyeloablative allografting in patients with T cell immunodeficiencies}

The investigators in Seattle hypothesized that patients with $\mathrm{T}$ cell immunodeficiencies would not require pre-transplant conditioning therapy and that marrow grafts would be able 
to create their own space. Post grafting, GVH and $\mathrm{HVG}$ reactions would be controlled solely with the use of CsA and MMF. Results of SCT from HLA-identical sibling donors in two patients with severe $\mathrm{T}$ cell immunodeficiency syndromes support the hypothesis. ${ }^{39}$ In both cases stable donor engraftment was established with varying proportions of myeloid and lymphoid engraftment. Corrections of the profound underlying immunodeficiencies were accomplished although as yet not completely. GVHD occurred early in both patients and was controlled with immunosuppressive drugs. One patient had chronic GVHD and died of sepsis and the other patient survives at 19 months with sustained engraftment, off immunosuppression and leading a normal life.

\section{Outpatient allografting in patients with malignancy}

The safety and efficacy of the canine pre-clinical studies helped develop a conceptual scheme of studies in patients with hematologic malignancy (Figure 1). Immunosuppression is divided into two components, one directed at host cells before the transplant, and the other at both donor and host cells after the transplant to provide simultaneous control of both GVH and HVG reactions. The goal is to establish bidirectional graft-host tolerance as manifested by stable mixed donor-host hematopoietic chimerism. This would establish an immunologic platform for the subsequent administration of DLI given specifically to eradicate the underlying malignancy.

The goal of these studies was to evaluate whether allogeneic hematopoietic cell engraftment could be established in patients with malignancies using peripheral blood stem cell grafts from HLA-identical donors using an immunosuppressive conditioning regimen of 200 cGy TBI pretransplant and short-term post-graft immunosuppression with $\mathrm{MMF}$ and $\mathrm{CsA} .{ }^{40} \mathrm{CsA}$ was given at $6.25 \mathrm{mg} / \mathrm{kg}$ p.o. twice daily from day -1 to day +35 or day +56 and targeted to blood levels at the upper end of the therapeutic range, with supratherapeutic levels tolerated in the absence of CsA toxicities. MMF was given at a dose of $15 \mathrm{mg}$ p.o. twice daily from day 0 to day +27 and discontinued without tapering. Eligibility for the study required a contraindication to the use of conventional allografting because of age, prior high-dose therapy, or organ dysfunction. Forty-six patients with a median age of 56 years (range $31-72$ ) were treated.
Diagnoses were AML $(n=11)$, CML $(n=8)$, CLL $(n=$ $8)$, multiple myeloma $(n=8)$, Hodgkin's disease $(n=4)$, non-Hodgkin's lymphoma $(n=3)$, acute lymphoblastic leukemia $(n=1)$, myelodysplastic syndrome $(n=1)$, breast cancer $(n=1)$, amyloidosis $(n=1)$. Thirty-two patients were eligible for outpatient allografting while the remainder were already hospitalized before transplant. Amongst those patients transplanted in an outpatient setting, the median number of days of subsequent hospitalization during the initial 60 days post transplant was 0 (range 0-26). Transplants were very well tolerated with mild myelosuppression, no development of mucositis and no additional alopecia. The only significant regimen-induced toxicities were reversible hepatoxicity in three patients. In two of these, predisposing factors were liver cirrhosis in one patient and concomitant amphotericin therapy in another. Of the fortysix patients treated, non-fatal graft rejection occurred in nine $(16 \%)$ patients. Four patients were not evaluable for sustained engraftment because of death from either transplant complications $(n=1)$ or early disease progression $(n$ $=3$ ). Spontaneous acute GVHD requiring treatment occurred in $36 \%$ of patients. Transplant-related deaths occurred in three patients $(6.5 \%)$.

With median follow-up of 160 (range 30-450) days, significant disease responses have been observed in the majority of patients with sustained engraftment after transplant. This has included molecular remissions in four CML patients and in two CLL patients and disappearance of paraprotein in four myeloma patients. Many of the patients are still being monitored for disease responses. Responses have frequently been gradual in onset occurring over a period of 4-12 months. This is an important difference from conventional allografting in which detection of disease post transplant usually represents failure of the treatment approach, and is potentially important in the development of optimal DLI strategies for use after nonmyeloablative transplants.

\section{High-intensity autotransplantation followed by immunosuppressive cytotoxic allotransplantation regimen}

Based on the efficacy and reduced toxicity of nonmyeloablative regimens, investigators in Genoa designed a combined protocol consisting of autografting followed by mini-allo-
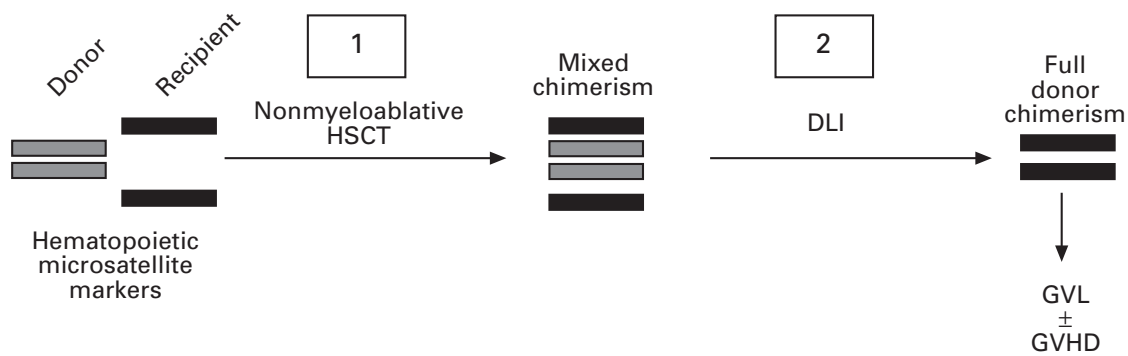

Figure 1 Conceptual scheme for non-myeloablative stem cell allografting. In step 1 stable mixed hematopoietic chimerism is established with immunosuppressive and minimally toxic conditioning therapy, thus minimizing regimen toxicities and GVHD with the initial transplant. This allows for step 2 , the infusion of donor lymphocytes without rejection, resulting in eradication of host hematopoiesis including the graft-versus-leukemia reaction. Newer strategies for minimizing GVHD with step 2 include the use of T cell dose escalation schemes, of CD8-depleted DLI, and the insertion of a suicide (eg herpes simplex thymidine kinase) gene into the $\mathrm{T}$ cells that can be activated by drugs. 
grafting for patients with advanced hematologic neoplasia and metastatic breast cancer. ${ }^{41,42}$ Thirty-two patients have been treated: 25 have follow-ups of over 3 months, and seven are within 45 days. Diagnoses in 25 evaluable patients were high-risk Hodgkin's disease (HD) $(n=10)$, non-Hodgkin's lymphoma (NHL) $(n=5)$, blastic (BP) or accelerated (AP) CML $(n=3)$, liver/bone metastatic breast cancer $(n=4)$. Patients with RAEB-t $(n=2)$ and AML $(n=1)$ received only mini-allografting. In preparation for autografting, the patients underwent high-dose therapy on protocols appropriate for the underlying disease. After engraftment of autologous stem cells, all patients were conditioned for allografting with fludarabine $30 \mathrm{mg} / \mathrm{m}^{2} /$ day $\times$ 3 days with cyclophosphamide $300 \mathrm{mg} / \mathrm{m}^{2} /$ day $\times 3$ days, designated the Flu-Cy protocol. Progenitor cells from HLAmatched donors were mobilized with G-CSF and infused to the patients. GVHD prophylaxis comprised CsA and methotrexate. After autografting, the results in the different diseases were as follows: NHL and HD: three complete remissions (CR), 11 partial remissions (PR) and one progressive disease; CML: AP: 1; BP: 2; breast cancer: all patients showed a reduction of pain but no changes in metastatic disease. After mini-allografting, complete chimerism was achieved in 15/25 (60\%) patients and mixed chimerism in seven $(30 \%)$ patients. Three patients (BP-CML, RAEB-t and AML) appear to have had autologous recovery. One patient with HD in remission after autografting maintained the remission after mini-allografting; two patients relapsed and are now alive in partial remission. Eight patients with NHL and HD, who obtained partial remission after autografting, achieved CR after mini-allografting and donor lymphocyte infusion. One patient with HD died while in CR of aspergillus infection in the brain and another patient with HD died of progressive disease combined with liver chronic GVHD. One patient (RAEB-t) achieved hematologic and cytogenetic remission and another patient (APCML) had hematologic and molecular remissions. Subsequently, the RAEB-t patient relapsed and she is now alive on day +520 after mini-allografting; the AP-CML patient is in continuous molecular remission at day +470 . One patient with breast cancer is alive in good partial remission (50\% reduction of bone metastasis) at day +600 . Grade III acute GVHD was the single major complication in nine patients; grade III acute GVHD was observed in two patients. In only two patients did the absolute neutrophil count decrease to $<1.0 \times 10^{9} / 1$. No patient required a sterile room. Five patients died of progressive disease. To date, $19 / 25$ evaluable patients are alive with a median followup from mini-allografting of 13 months (range 3-26). In summary, immunosuppressive therapy with Flu-Cy allowed engraftment of hematopoietic progenitor cells from HLAmatched sibling donors; only one patient died of the procedure 120 days after mini-allografting.

\section{Conclusions}

While periods of follow-up are still short in the patients described above, this new approach has dramatically reduced the acute toxicity of allografting even among patients who would otherwise have been excluded from conventional allografting because of age or other high risk factors. These new less toxic procedures should facilitate future studies of adoptive immunotherapy for malignancies and allow us to extend the use of this type of allografting to the treatment of selected non-malignant diseases.

\section{References}

1 Thomas ED. The role of bone marrow transplantation for eradication of malignant disease. Cancer 1969; 10: 19631969.

2 Thomas ED. Bone marrow transplantation for malignant disease. J Clin Oncol 1983; 1: 517-531.

3 Rondòn G, Giralt S, Huh Y et al. Graft-versus-leukemia effect after allogeneic bone marrow transplantation for chronic lymphocytic leukemia. Bone Marrow Transplant 1996; 18: 669672.

4 Van Besien K, Sobocinski K, Rowlings PA et al. Allogeneic bone marrow transplantation for low-grade lymphoma. Blood 1998; 92: 1832-1836.

5 Khouri IF, Przepiorka D, Van Besien K et al. Allogeneic blood or marrow transplantation for chronic lymphocytic leukaemia: timing of transplantation and potential effect of fludarabine on acute graft-versus-host disease. $\mathrm{Br} J$ Haematol 1997; 97: 466-473.

6 Tricot G, Vesole DH, Jagannath S et al. Graft-versus-myeloma effect: proof of principle. Blood 1996; 87: 1196-1198.

7 Ueno NT, Rondòn G, Mirza NQ et al. Allogeneic peripheralblood progenitor-cell transplantation for poor-risk patients with metastatic breast cancer. J Clin Oncol 1998; 16: 986993.

8 Eibl B, Schwaighofer H, Nachbaur D et al. Evidence for a graft-versus-tumor effect in a patient treated with marrow ablative chemotherapy and allogeneic bone marrow transplantation for breast cancer. Blood 1996; 88: 1501-1508.

9 Childs RW, Clave E, Tisdale J et al. Successful treatment of metastatic renal cell carcinoma with a non-myeloablative allogeneic peripheral-blood progenitor-cell transplant: evidence for a graft-versus-tumor effect. J Clin Oncol 1999; 17: 2044-2050.

10 Slavin S, Naparstek E, Nagler A et al. Allogeneic cell therapy for relapsed leukemia following bone marrow transplantation with donor peripheral blood lymphocytes. Exp Hematol 1995; 23: $1553-1562$.

11 Slavin S, Naparstek E, Nagler A et al. Allogeneic cell therapy with donor peripheral blood cells and recombinant human interleukin-2 to treat leukemia relapse post allogeneic bone marrow transplantation. Blood 1996; 87: 2195-2204.

12 Kolb HJ, Mittermuller J, Clemm C et al. Donor leukocyte transfusions for treatment of recurrent chronic myelogenous leukemia in marrow transplant patients. Blood 1990; 76: 2462-2465.

13 Porter DL, Roth MS, McGarigle C et al. Induction of graftversus-host disease as immunotherapy for relapsed chronic myeloid leukemia. New Engl J Med 1994; 330: 100-106.

14 Kolb HJ, Schattenberg A, Goldman JM et al. Graft-versusleukemia effect of donor lymphocyte transfusions in marrow grafted patients: European Group for Blood and Marrow Transplantation Working Party Chronic Leukemia. Blood 1995; 86: 2041-2050.

15 Mackinnon S, Papadopoulos EB, Carabassi MH et al. Adoptive immunotherapy evaluating escalating doses of donor leukocytes for relapse of chronic myeloid leukemia after bone marrow transplantation: separation of graft-versus-leukemia responses from graft-versus-host disease. Blood 1995; 86: $1261-1268$ 
16 Giralt S, Hester J, Huh Y et al. CD8-depleted donor lymphocyte infusions as treatment for relapsed chronic myelogenous leukemia after allogeneic bone marrow transplantation. Blood 1995; 86: 4337-4343.

17 Giralt S, Gajewski J, Khouri I et al. Induction of graft-versusleukemia as primary treatment of chronic myelogenous leukemia. Blood 1997; 90: 3204-3213.

18 Collins RH Jr, Shpilberg O, Drobyski WR et al. Donor lymphocyte infusions in 140 patients with relapsed malignancy after allogeneic bone marrow transplantation. J Clin Oncol 1997; 15: 433-444.

19 Antin JH, Ferrara JLM. Cytokine dysregulation and acute graft-versus-host disease. Blood 1992; 80: 2964-2968.

20 Storb R, Yu C, Barnett T et al. Stable mixed hematopoietic chimerism in DLA-identical littermate dogs given lymph node irradiation before and pharmacological immunosuppression after marrow transplantation. Blood 1999; 90: 1131-1136.

21 Giralt S, Estey E, Albitar M et al. Engraftment of allogeneic hematopoietic progenitor cells with purine analog-containing chemotherapy: harnessing graft-versus-leukemia without myeloablative therapy. Blood 1997; 89: 4531-4536.

22 Slavin S, Nagler A, Naparstek E et al. Nonmyeloablative stem cell transplantation and cell therapy as an alternative to conventional bone marrow transplantation with lethal cytoreduction for the treatment of malignant and nonmalignant hematologic diseases. Blood 1998; 91: 756-763.

23 Keating MJ, Kantarjian H, Talpaz M et al. Fludarabine a new agent with major activity against chronic lymphocytic leukemia. Blood 1989; 74: 19-25.

24 Storb R. Nonmyeloablative preparative regimens: experimental data and clinical practice. ASCO: 1999 Educational Book, p 241, 55th Annual Meeting, May 15-18, Atlanta.

25 Giralt S, Gajewski J, Khouri I et al. Induction of graft-versusleukemia as primary treatment of chronic myelogenous leukemia. Blood 1997; 90 (Suppl.1): 1857a.

26 Giralt S, Cohen A, Mehra R et al. Preliminary results of fludarabine/melphalan or $2 \mathrm{CDA} /$ melphalan as preparative regimens for allogeneic progenitor cell transplantation in poor candidates for conventional myeloablative conditioning. Blood 1997; 90 (Suppl.1): 1853a.

27 Khouri IF, Keating M, Korbling M et al. Transplant-lite: induction of graft-versus-malignancy using fludarabine-based nonablative chemotherapy and allogeneic blood progenitorcell transplantation as treatment for lymphoid malignancies. $J$ Clin Oncol 1998; 16: 2817-2824.

28 Childs R, Clave E, Contentin N et al. Engraftment kinetics after nonmyeloablative allogeneic peripheral blood stem cell transplantation: full donor T-cell chimerism precedes alloimmune responses. Blood 1999; 94: 3234-3241.

29 Sykes M, Preffer F, McAfee S et al. Mixed lymphohematopoietic chimerism and graft versus lymphoma effect are achievable in adult recipients following non-myeloablative therapy and HLA-mismatched donor bone marrow transplantation. Lancet 1999; 353: 1755-1759
30 Slavin S, Nagler A, Naparstek E et al. Nonmyeloablative stem cell transplantation and cell therapy as an alternative to conventional bone marrow transplantation with lethal cytoreduction for the treatment of malignant and nonmalignant hematologic diseases. Blood 1998; 91: 756-763.

31 Nagler A, Or R, Naparstek E et al. Matched unrelated bone marrow transplantation (BMT) using a non-myeloablative conditioning regimen. Blood 1998; 92 (Suppl. 1): 289a (Abstr. 1185).

32 Nagler A, Or R, Naparstek E et al. Secondary allogeneic stem cell transplantation (alloSCT) using a non-myeloablative conditioning regimen for patients with hematological malignancies. Blood 1998; 92 (Suppl. 1): 137a (Abst. 553).

33 Wagner JL, Storb R. Preclinical large animal models for hematopoietic stem cell transplantation. Curr Opin Hematol 1996; 3: $410-416$.

34 Storb R, Raff RF, Graham T et al. Marrow toxicity of fractionated versus single dose total body irradiation is identical in a canine model. Int J Radiat Oncol Biol Phys 1993; 26: 275281.

35 Storb R, Raff RF, Appelbaum FR et al. Comparison of fractionated to single-dose total body irradiation in conditioning canine littermates for DLA-identical marrow grafts. Blood 1989; 74: 1139-1143.

$36 \mathrm{Yu}$ C, Storb R, Mathey B et al. DLA-identical bone marrow grafts after low-dose total body irradiation: effects of highdose corticosteroids and cyclosporine on engraftment. Blood 1995; 86: 4376-4381.

37 Storb R, Yu C, Wagner JL et al. Stable mixed hematopoietic chimerism in DLA-identical littermate dogs given sublethal total body irradiation before and pharmacological immunosuppression after marrow transplantation. Blood 1997; 89: 3048-3054

38 Storb R, Yu C, Zaucha JM et al. Stable mixed hematopoietic chimerism in dogs given donor antigen, CTLA4Ig, and 100 cGy TBI before and pharmacological immunosuppression after marrow transplant. Blood 1999; 94: 2523-2529.

39 Woolfrey AE, Nash RA, Frangoul HA et al. Non-myeloablative transplant regimen used for induction of multi-lineage allogeneic hematopoietic mixed donor-host chimerism in patients with T-cell immunodeficiency. Blood 1998; 92 (Suppl. 1): 520a (Abstr. 2135).

40 McSweeney PA, Storb R. Mixed chimerism: preclinical studies and clinical applications. Biol Blood Marrow Transplant 1999; 5: 192-203.

41 Carella AM, Lerma E, Dejana A et al. Engraftment of HLAmatched sibling hematopoietic stem cells after immunosuppressive conditioning regimen in patients with hematologic neoplasias. Haematologica 1998; 83: 904-909.

42 Carella AM, Lerma E, Corsetti MT et al. Evidence of cytogenetic and molecular remission by allogeneic cells after immunosuppressive therapy alone. Br J Haematol 1998; 103: $565-567$. 